\title{
Surface Reconstruction from Gradient Fields via Gradient Transformations
}

\author{
Amit Agrawal · Ramesh Raskar • Rama Chellappa
}

Received: 7 October 2008 / Accepted: 22 September 2009

(C) Springer Science+Business Media, LLC 2009

This article has been published Online First, but is withdrawn at the request of the authors.

\footnotetext{
A. Agrawal (凶)

Mitsubishi Electric Research Labs (MERL), 201 Broadway,

Cambridge, MA 02139, USA

e-mail: agrawal@merl.com

R. Raskar

MIT Media Lab, Cambridge, MA 02139, USA

e-mail: raskar@media.mit.edu

R. Chellappa

Dept. of Electrical and Computer Engineering, Center for Automation Research, UMIACS, University of Maryland,

College Park, MD 20742, USA

e-mail:rama@umiacs.umd.edu
} 